\title{
Magneto-optical determination of the electron-solid phase boundary
}

\author{
M. K. Ellis, M. Hayne, A. Usher, and A. S. Plaut \\ Department of Physics, University of Exeter, Stocker Road, Exeter EX4 4QL, United Kingdom \\ K. Ploog \\ Max-Planck-Institut für Festkörperforschung, D-7000 Stuttgart 80, Federal Republic of Germany
}

(Received 11 June 1991; revised manuscript received 3 April 1992)

\begin{abstract}
We have obtained a two-dimensional electron-solid phase diagram in the extreme magnetic quantum limit by studying the temperature dependence of the radiative recombination of electrons in a $\mathrm{GaAs} / \mathrm{Al}_{x} \mathrm{Ga}_{1-x} \mathrm{As}$ heterojunction with holes bound to a $\delta$ layer, $250 \AA$ away in the $\mathrm{GaAs}$, of $\mathrm{Be}$ acceptors. The low-energy shoulder to the luminescence line, indicating the presence of the electron solid, is seen to disappear at a filling-factor-dependent critical temperature. We observe no shoulder above a filling factor of 0.25 , and the critical temperature falls to below $0.4 \mathrm{~K}$ at filling factors $\frac{1}{5}$ and $\frac{1}{7}$.
\end{abstract}

The fractional quantum Hall effect (FQHE) occurs as a result of the condensation, in the extreme magnetic quantum limit, of a two-dimensional (2D) electron gas into an incompressible Fermi-liquid ground state. ${ }^{1-3}$ This occurs at fractional filling factors $v=h n_{s} / e B=p / q$, where $p$ and $q$ are integers with $q$ odd, $n_{s}$ is the 2D electron concentration, and $B$ is the perpendicular magneticfield strength. The first observations of the FQHE quantum fluid came from magnetotransport measurements which detected plateaus in the Hall resistance (Ref. 2 and references therein). However, at high magnetic fields and low temperatures these transport studies are very sensitive to magnetic localization effects leading to a very high background resistivity and consequent problems with electrical noise. ${ }^{4-6}$ In contrast, magneto-optical studies in the FQHE regime do not suffer from these problems and are therefore particularly useful for investigations at very small $v$. The first optical measurements in this regime were made on silicon metal-oxide-semiconductor fieldeffect transistors; ${ }^{7}$ subsequently, there have been a number of studies using $\mathrm{GaAs} / \mathrm{Al}_{x} \mathrm{Ga}_{1-x}$ As structures. ${ }^{8-11}$ Buhmann et al., ${ }^{9}$ using beryllium $\delta$-doped GaAs/ $\mathrm{Al}_{x} \mathrm{Ga}_{1-x} \mathrm{As}$ heterojunctions, see fractional states down to a filling factor of $\frac{1}{9}$ which they detect as discontinuities in the energy peak position as a function of magnetic field. Energy shifts, line splittings, and intensity minima at fractions are also reported in optical investigations by Goldberg et al. ${ }^{10}$ and Turberfield et al. ${ }^{11}$

Calculations using the Laughlin variational wave function $^{3}$ for the ground and excited states of the twodimensional electron system (2DES) predict a phase transition from the Fermi-liquid state to the Wigner crystal at filling factor $v \approx \frac{1}{10}$. Other theoretical calculations ${ }^{12-14}$ have suggested critical values of filling factor $v_{c}$ between $\frac{1}{10}$ and $\frac{1}{3}$. Lam and Girvin, ${ }^{12}$ in particular, make a substantial improvement over previous Hartree-Fock calculations ${ }^{14}$ by using a variational wave function which includes particle correlations. They find a critical filling factor for Wigner solidification given by $v^{-1}=6.5 \pm 0.5$. In order to investigate this phase transition, magnetotransport experiments have been carried out by Willett $e t$ $a l .{ }^{5}$ on high-mobility samples down to $v \approx \frac{1}{18}$. Several features of their results are consistent with the presence of a pinned Wigner solid; in particular, they see an activated temperature dependence of the conduction below $v=0.27$. Andrei et al. ${ }^{15}$ have obtained a critical filling factor for Wigner crystallization of 0.23 from the results of radiofrequency measurements. However, measurements of the quantized Hall resistance and minima in the Shubnikovde Haas oscillations have shown that the ground state at $v=\frac{1}{5}$ is still the quantum liquid, ${ }^{5,6,16}$ and not the electron solid. Goldman, Shayegan, and Tsui ${ }^{6}$ also report evidence for the state with $v=\frac{1}{7}$ being the Laughlin liquid. The experiments of Jiang et al. ${ }^{16}$ have shown a diagonal resistivity in the vicinity of $v=\frac{1}{5}$ that diverges exponentially as the temperature is reduced. This is in sharp contrast to the diagonal resistivity seen at exactly $v=\frac{1}{5}$ which vanishes as the temperature is lowered, and indicates a reentrant solid phase near the fraction. Studies of threshold conduction ${ }^{17}$ have also demonstrated this reentrant behavior of the pinned electron-solid phase around $v=\frac{1}{5}$. It is important to note, however, that the theoretical studies mentioned above only find a single transition from the liquid to the solid. Buhmann et al. ${ }^{18}$ have recently carried out the first optical investigations in the Wigner solid regime. Their luminescence spectra show, at low temperatures and below a critical filling factor $\left(v_{c}=0.28\right)$, a new line appearing as a low-energy shoulder to the main Landau-level luminescence peak. The shoulder grows in intensity as $v$ decreases and eventually dominates the spectrum at fields above $25 \mathrm{~T}$ where the filling factor is less than $\frac{1}{11}$. Importantly, they also observe a weakening of the shoulder at fractional filling factors $v=\frac{1}{5}, \frac{1}{7}$, and $\frac{1}{9}$, in agreement with the above transport experiments. These dependences on filling factor and temperature, observed at a number of electron concentrations, constitute compelling evidence for the low-energy shoulder being associated with the formation of the electron solid. ${ }^{18}$ Thus the present state of our knowledge of the electron solid, as obtained from the above experiments, is that it occurs below a critical filling factor of approximately 0.25 , and that there is competition between the electron solid and 
Laughlin liquid states around the fractional filling factors $v=\frac{1}{5}$ and $\frac{1}{7}$. Although Buhmann et al. ${ }^{18}$ speculate as to the qualitative form of the phase diagram, no experimental phase diagram has been published; we present such a diagram in this paper.

Here we report the results of luminescence studies of a heterojunction similar to that used by Buhmann et al. ${ }^{18}$ The molecular-beam-epitaxy-grown sample is a GaAs/ $\mathrm{Al}_{x} \mathrm{Ga}_{1-x} \mathrm{As}$ heterojunction with a $\delta$ layer of Be acceptors in the GaAs situated $250 \AA$ from the interface. The radiative transition detected in these experiments results from recombination between the $2 \mathrm{DES}$ and holes bound to the Be acceptors. ${ }^{19}$ We have performed a systematic investigation of the temperature and magnetic-field dependences of the intensity of the low-energy shoulder which is associated with electron solidification, and from this we obtain the electron-solid phase diagram in this regime. Experiments were carried out in a ${ }^{3} \mathrm{He}$ cryostat with optical access via a single optical fiber. Measurements were made in fields up to $17 \mathrm{~T}$ and temperatures down to $0.4 \mathrm{~K}$; thermometry consisted of a germanium resistor and a ruthenium-oxide chip resistor with negligible magnetoresistance. Luminescence and magnetotransport studies of the effects of photoexcitation on these 2D systems have been reported by Kukushkin et al. ${ }^{19}$ They have shown that, for the structure studied here, continuous illumination with argon-ion laser light causes the sheet electron concentration $\left(n_{s}\right)$ to be reduced. With an incident power of $10^{-2} \mathrm{~W} \mathrm{~cm} n_{s}$ reaches a value of $4.7 \times 10^{10} \mathrm{~cm}^{-2}$, and any further increase in intensity does not change $n_{s}$ significantly. Confirmation of this value of $n_{s}$ under the conditions of the optical experiment was obtained by low-field Hall measurements (see inset in Fig. 3), during which the whole of the Hall bar was illuminated. The illumination conditions of our experiments are sufficient to obtain the above value of $n_{s}$ but the presence of bulk conduction in the sample then prevents high-field magnetotransport measurements. At all illumination levels there was no noticeable dependence of $n_{s}$ on magnetic field. We have confirmed this lack of field dependence of the concentration by carrying out Shubnikov-de Haas measurements at lower light levels where agreement was found between the concentration obtained from the Hall coefficient, from the position of the minima in the Shubnikov-de Haas oscillations and from simultaneous luminescence measurements. It should be noted that the lack of field dependence of the electron concentration in acceptor $\delta$-doped samples is in marked contrast to the behavior of modulation-doped heterojunctions ${ }^{11}$ which seem to display a magnetic-field-dependent depletion of the 2D electron gas. ${ }^{20}$ It is clear, therefore, that the depletion mechanisms in the two cases must be quite different.

At high magnetic fields $(\geq 9 \mathrm{~T})$ and low temperatures $(\leq 1.3 \mathrm{~K})$, a shoulder is seen on the low-energy side of the main luminescence peak. This feature is found, in general, to increase in intensity as the field is increased and as the temperature is lowered. However, at the fractional filling factors $v=\frac{1}{5}$ and $\frac{1}{7}$ a reduction in the intensity of the low-energy shoulder is observed, similar to that reported by Buhmann et al. ${ }^{18}$ This weakening of the shoulder is clearly visible in the raw data as shown in Fig.
1. Here we present the luminescence spectra taken at 0.9 $\mathrm{K}$ for three values of magnetic field: $12.5,14.5$, and 16.5 T. The 14.5-T spectrum corresponds closely to $v=\frac{1}{7}$ fractional filling. In each of the three graphs the solid curve is a high-temperature luminescence spectrum, taken at the same magnetic field, with width adjusted for thermal broadening and height matched to fit the lowtemperature data, and is used for comparison purposes. It is clearly seen that the relative shoulder intensity has a smaller value in the 14.5-T spectrum, with an increase in intensity at both lower and higher fields. This behavior is indicative of the competition between the electron solid and the incompressible quantum fluid as the ground state for this system. In order to produce an electron-solid phase diagram a quantitative analysis of the low-energy shoulder behavior has been carried out as described below.

It is necessary to analyze the height of the low-energy shoulder by a method which distinguishes it from the main luminescence peak, even if the latter is changing in width and height. The luminescence line shape is asymmetric and so we have used a method in which the line shape of each spectrum is compared with one taken in conditions under which there is no low-energy shoulder,
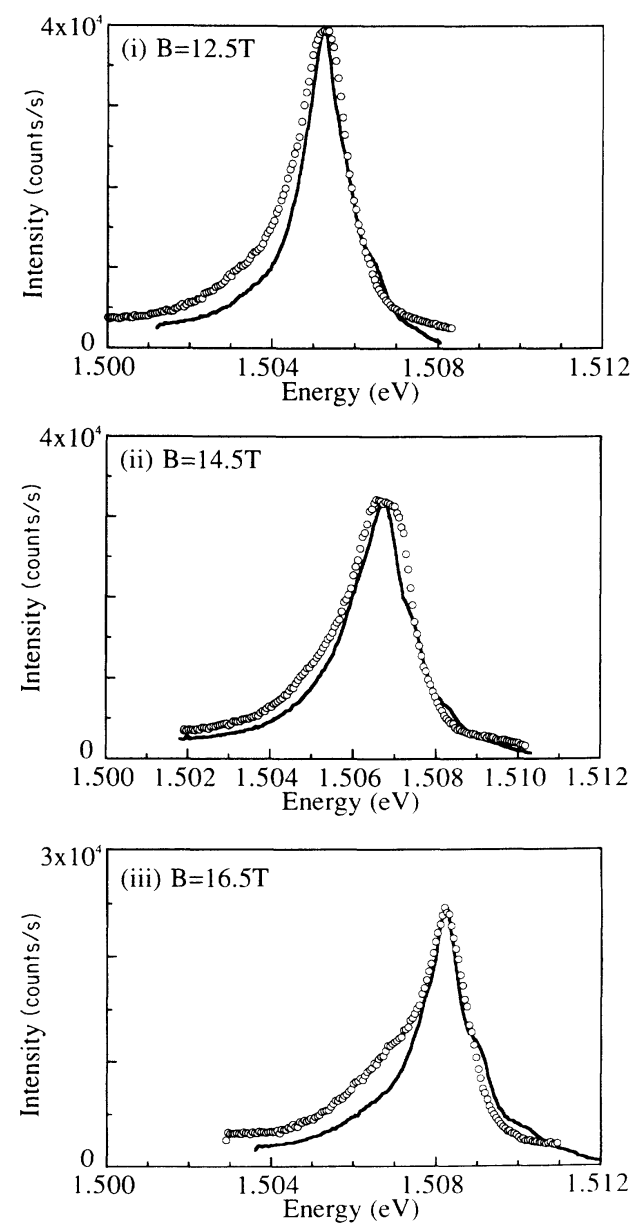

FIG. 1. Luminescence spectra taken at $0.9 \mathrm{~K}$ (open circles) for $B=12.5,14.5$, and $16.5 \mathrm{~T}$. Normalized data for $4.25 \mathrm{~K}$ are also shown for comparison (solid lines). 
along with the reasonable assumption that thermal broadening of the luminescence line is independent of field. We have made this comparison using hightemperature spectra as follows: (i) at low field, away from the electron-solid regime (but below $v=1$ ), the lowtemperature spectrum is compared with one taken at the same field, but at high temperature. The ratio of the widths of these two spectra then gives the relative thermal broadening which is assumed to be independent of field; (ii) at higher fields and lower temperatures, where the shoulder is present, the spectrum under consideration is compared with that at high temperature, but at the same field; (iii) the difference between these spectra, after the thermal broadening obtained above has been taken into account and the high-temperature line been scaled to match the low-temperature peak intensity, then gives the size of the low-energy shoulder. In order to enable comparison of different data sets the strength of the shoulder is expressed as a ratio of the shoulder intensity to the peak intensity of the main luminescence line.

This technique, although complicated, is nevertheless thorough: it involves a comparison of spectra taken at the same fields, eliminating concern about the change of line shape with field, and it also takes account of temperature effects by making the reasonable assumption that thermal broadening is independent of field. The typical temperature dependence of the shoulder intensity ratio, at a fixed field of $16.5 \mathrm{~T}$, is shown in Fig. 2. It can be seen that there is a transition at a critical temperature between a pronounced shoulder at lower temperatures, and a reduced one at higher temperatures. Such behavior is suggestive of the melting of the electron solid. It should be noted that we have used other analysis techniques, such as comparison with low-field spectra at the same temperature, and although we regard them as less suitable than the above method they still produce the same critical dependence of the shoulder intensity on temperature. This independence of the results on the exact method of analysis further reinforces our conclusions. In Fig. 1 the solid lines, derived from high-temperature spectra, show the emergence of features on the high-energy side which are a result of thermal population of higher-energy hole states. However, since these lines are weak and lie on the high-energy side of the main line they make no contribution to the shoulder intensity.

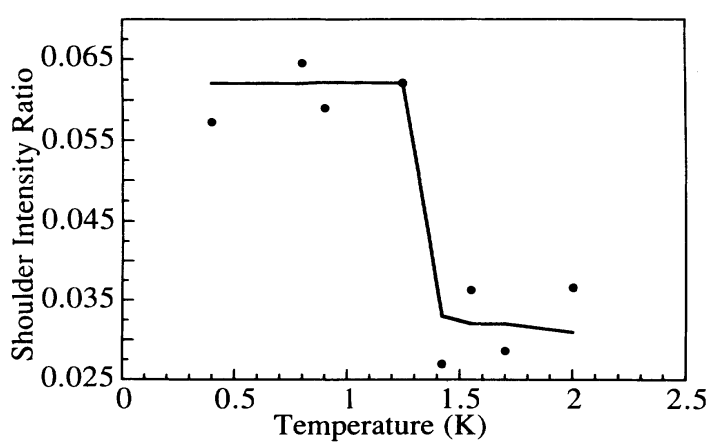

FIG. 2. Shoulder intensity ratio vs temperature for $B=16.5$ T. The solid line is a guide to the eye.

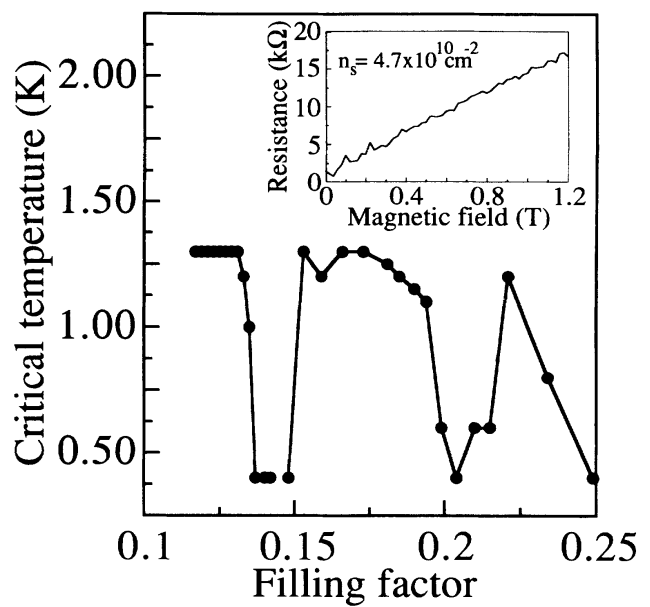

FIG. 3. Electron-solid phase diagram. The low-field Hall measurement is shown as an inset.

The solid-phase critical temperature, which is taken to be the point at which the shoulder intensity falls to halfway between its value on either side of the transition shown in Fig. 2, is plotted against the filling factor in Fig. 3. It is found to decrease, falling below the lowest experimental temperature, at fractional filling factors, as seen qualitatively in the spectra of Fig. 1. This variation in the shoulder intensity is indicative of the melting of the electron solid at fractional filling factors, and is consistent with the results of experiments mentioned in the introduction. 5,6,16,17 Furthermore, this solid phase diagram shows that there is a decrease in the critical temperature with increasing filling factor until the Wigner crystal is no longer present above $v=0.25$ at any temperature.

We have, in addition, observed a field-dependent broadening of the main luminescence line, persistent to temperatures above those at which the low-energy shoulder disappears. Figure 4 shows the ratio of the low-energy half-widths at $2 \mathrm{~K}$ to those at $4.25 \mathrm{~K}$, both temperatures at which there is no low-energy shoulder. At low fields the ratio of linewidths is constant and just below one, as expected. However, above $9 \mathrm{~T}$ the lower-temperature line is seen to broaden with increasing field relative to that at higher temperature. We attribute this broadening to

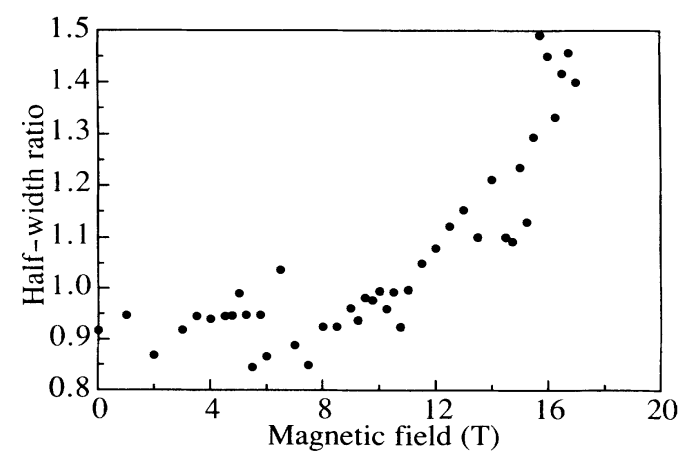

FIG. 4. Ratio of low-energy half-widths at $2 \mathrm{~K}$ to those at $4.25 \mathrm{~K}$ vs magnetic field. 
magnetic localization of the electron states in the lowenergy tail of the Landau level. Although the exact shape of a disorder-broadened Landau level is dependent on the amount and type of disorder present it is known that, in a system such as that described here, disorder gives rise to distinct localized- and extended-states regions, ${ }^{21-24}$ the former occurring in the Landau-level tails. At relatively low magnetic fields the narrow extended-state region in the center of the Landau level is responsible for most of the observed luminescence, the localized states contributing only a little to the intensity. However, at higher fields the Fermi energy lies in the low-energy tail of the Landau level and consequently the luminescence, now weaker, must come from the localized electrons in these tails. The fact that the luminescence broadens with field therefore demonstrates that the localized-state tail is wide compared with the extended-state region. One consequence of the broadening of the luminescence line by these magnetically localized states is an additional contribution to the shoulder intensity as determined by the present method of analysis. This explains the presence of a residual shoulder intensity ratio both above the critical temperature and also at fractional filling factors (as illustrated in Fig. 1) where no critical temperature dependence is observed. The gradual nature of the broadening of the luminescence caused by disorder-induced localization makes this effect readily distinguishable from the sudden appearance of a new line, which is first seen as a low-energy shoulder, but eventually dominates the luminescence spectrum and has been identified as a signature of the $2 \mathrm{D}$ electron solid. ${ }^{18}$

In summary, we have measured the filling factor dependence of the critical temperature of the low-energy shoulder in the 2DES luminescence, appearing below $v=0.25$, which is associated with the formation of the electron solid, and have thus produced an electron-solid phase diagram. At fractional filling factors $\frac{1}{5}$ and $\frac{1}{7}$ the critical temperature is below the lowest experimental temperature which indicates that the ground state at these fractions is the incompressible electron liquid, in agreement with previous experiments. We see a broadening of the main luminescence line at high fields which persists above the temperature at which the low-energy shoulder disappears. We attribute this effect to disorder-induced magnetic localization. Our results thus enable us to distinguish between an intrinsic effect (electron solidification) and one with extrinsic origin in the sample disorder (magnetic localization).

Note added. Recent work by Kukushkin et al. ${ }^{25}$ has suggested that an intermediate solid-liquid phase may exist. Our data show the low-energy shoulder coexisting with the main luminescence line due to the Laughlin liquid and its quasiparticle excitations and are therefore consistent with the possible existence of an intermediate phase.
'D. C. Tsui, H. L. Stormer, and A. C. Gossard, Phys. Rev. Lett. 48, 1559 (1982).

${ }^{2}$ For a review see T. Chakraborty and P. Pietiläinen, The Fractional Quantum Hall Effect (Springer-Verlag, New York, 1988).

${ }^{3}$ R. B. Laughlin, Phys. Rev. Lett. 50, 1395 (1983).

${ }^{4}$ E. E. Mendez, L. L. Chang, M. Heiblum, L. Esaki, M. Naughton, K. Martin, and J. Brooks, Phys. Rev. B 30, 7310 (1984).

${ }^{5}$ R. L. Willett, H. L. Stormer, D. C. Tsui, L. N. Pfeiffer, K. W. West, and K. W. Baldwin, Phys. Rev. B 38, 7881 (1988).

${ }^{6}$ V. J. Goldman, M. Shayegan, and D. C. Tsui, Phys. Rev. Lett. 61, 881 (1988).

${ }^{7}$ I. V. Kukushkin and V. B. Timofeev, Pis'ma Zh. Eksp. Teor. Fiz. 44, 179 (1986) [JETP Lett. 44, 228 (1986)].

${ }^{8}$ D. Heiman, B. B. Goldberg, A. Pinczuk, C. W. Tu, A. C. Gossard, and J. H. English, Phys. Rev. Lett. 61, 605 (1988).

${ }^{9}$ H. Buhmann, W. Joss, K. von Klitzing, I. V. Kukushkin, G. Martinez, A. S. Plaut, K. Ploog, and V. B. Timofeev, Phys. Rev. Lett. 65, 1056 (1990).

${ }^{10}$ B. B. Goldberg, D. Heiman, A. Pinczuk, L. Pfeiffer, and K. W. West, Phys. Rev. Lett. 65, 641 (1990).

${ }^{\prime \prime}$ A. J. Turberfield, S. R. Haynes, P. A. Wright, R. A. Ford, R. G. Clark, J. F. Ryan, J. J. Harris, and C. T. Foxon, Phys. Rev. Lett. 65, 637 (1990).

12P. K. Lam and S. M. Girvin, Phys. Rev. B 30, 473 (1984).

${ }^{13}$ D. Levesque, J. Weiss, and A. H. MacDonald, Phys. Rev. B 30, 1056 (1984); S. T. Chui, T. M. Hakim, and K. B. Ma, ibid. 33, 7110 (1986); S. Kivelson, C. Kallin, D. P. Arovas, and J. R. Schrieffer, ibid. 36, 1620 (1987).
${ }^{14}$ D. Yoshioka and P. A. Lee, Phys. Rev. B 27, 4986 (1983).

${ }^{15}$ E. Y. Andrei, G. Deville, D. C. Glattli, F. 1. B. Williams, E. Paris, and B. Etienne, Phys. Rev. Lett. 60, 2765 (1988).

${ }^{16}$ H. W. Jiang, R. L. Willett, H. L. Stormer, D. C. Tsui, L. N. Pfeiffer, and K. W. West, Phys. Rev. Lett. 65, 633 (1990).

${ }^{17}$ V. J. Goldman, M. Santos, M. Shayegan, and J. E. Cunningham, Phys. Rev. Lett. 65, 2189 (1990); H. W. Jiang, H. L. Stormer, D. C. Tsui, L. N. Pfeiffer, and K. W. West, Phys. Rev. B 44, 8107 (1991).

${ }^{18}$ H. Buhmann, W. Joss, K. von Klitzing, I. V. Kukushkin, G. Martinez, A. S. Plaut, K. Ploog, and V. B. Timofeev, Phys. Rev. Lett. 66, 926 (1991).

${ }^{19} 1$. V. Kukushkin, A. S. Plaut, K. von Klitzing, and K. Ploog, Surf. Sci. 229, 447 (1990).

${ }^{20}$ R. G. Clark, R. A. Ford, S. R. Haynes, J. F. Ryan, A. J. Turberfield, P. A. Wright, C. T. Foxon, and J. J. Harris, in High Magnetic Fields in Semiconductors III, edited by G. Landwehr (Springer-Verlag, Berlin, 1992), p. 231.

${ }^{21}$ J. P. Eisenstein, H. L. Stormer, V. Narayanamurti, A. Y. Cho, A. C. Gossard, and C. W. Tu, Phys. Rev. Lett. 55, 875 (1985).

${ }^{22}$ E. Gornik, R. Lassnig, G. Strasser, H. L. Störmer, A. C. Gossard, and W. Wiegmann, Phys. Rev. Lett. 54, 1820 (1985).

${ }^{23}$ E. Stahl, D. Weis, G. Weimann, K. von Klitzing, and K. Ploog, J. Phys. C 18, L783 (1985).

${ }^{24}$ M. S. Skolnick, K. J. Nash, S. J. Bass, P. E. Simmonds, and M. J. Kane, Solid State Commun. 67, 637 (1988).

${ }^{25}$ I. V. Kukushkin, N. J. Pulsford, K. von Klitzing, K. Ploog, and V. B. Timofeev, Surf. Sci. 263, 30 (1992). 